QUARTERLY OF APPLIED MATHEMATICS

VOLUME LXX, NUMBER 4

DECEMBER 2012, PAGES 685-703

S 0033-569X(2012)01276-X

Article electronically published on June 20, 2012

\title{
EFFICIENT NUMERICAL STABILITY ANALYSIS OF DETONATION WAVES IN ZND
}

\author{
BY \\ JEFFREY HUMPHERYS (Department of Mathematics, Brigham Young University, Provo, Utah \\ 84602) \\ AND
}

KEVIN ZUMBRUN (Department of Mathematics, Indiana University, Bloomington, Indiana 47402)

Abstract. As described in the classic works of Lee-Stewart and Short-Stewart, the numerical evaluation of linear stability of planar detonation waves is a computationally intensive problem of considerable interest in applications. Reexamining this problem from a modern numerical Evans function point of view, we derive a new algorithm for their stability analysis, related to a much older method of Erpenbeck, that, while equally simple and easy to implement as the standard method introduced by Lee-Stewart, appears to be potentially faster and more stable.

1. Introduction. As described for example in 20, 21, 22, 34, 44, 45, the numerical stability analysis of detonation wave solutions of the Zeldovich-von Neumann-Döring (ZND), or reactive Euler equations, is a rich and computationally challenging problem. Planar detonation waves can often change stability as physical parameters are varied, undergoing interesting bifurcations to pulsating, spinning, and cellular solutions [12, 23, 2, 32, 35, 29, 47, 48, 49. This motivates the numerical study of their stability, originated by Erpenbeck in [20, 21], both for its interest in its own right and as a benchmark for more general time-evolution codes [12, 45].

Due both to the number of physical parameters (four for a polytropic ga: 1 ) and the difficulty of individual computations, this problem has proven to be numerically intensive. In their classical 1990 paper [34, in which they introduced the algorithm that has become the modern-day standard, computing accurately for the first time the stability

Received November 6, 2010.

2000 Mathematics Subject Classification. Primary 76E99; Secondary 76L05.

This work was supported in part by the National Science Foundation award numbers DMS-0607721 and DMS-0300487, and National Science Fountation CAREER award DMS-0847074. Thanks to Mark Williams for stimulating discussions regarding the numerical literature on stability of ZND detonations. E-mail address: jeffh@math.byu.edu

E-mail address: kzumbrun@indiana.edu

${ }^{1}$ Gas constant $\Gamma=\gamma-1$, heat release coefficient $q$, activation energy $E_{A}$, and detonation amplitude [20. 34] [55].

(C) 2012 Brown University

Reverts to public domain 28 years from publication 
boundaries for one-dimensional detonations, Lee and Stewart conclude (p. 131 of the reference): "Finally, we point out that though our scheme is direct and easy to implement, complete investigation of the various regions of parameter space is computationally intensive. Any equivalent or more efficient numerical method should be considered a valuable contribution and such approaches are needed to further explore the parameter regimes of instability."

Despite these comments, the basic algorithm introduced by Lee and Stewart (or perhaps variants thereof) as described in the 2006 survey 45 appears still to be the current state of the art. Of course, computational power has increased tremendously in the interim, making once-prohibitive computations now accessible. Nonetheless, it seems of interest to explore more efficient algorithms if they can be found.

In particular, the computations of [34] were carried out in 1990 on a Cray X-MP/48 supercomputer 2 with several hours required to produce individual figures. (For example, Fig. 9 of [34] tracking the top 6 unstable eigenvalues of detonations of a polytropic gas with gas constant $\gamma=1.2$ as activation energy is varied was reported to require 5 hours of computation.) Today, substantially more computing power is available in a standard desktop PC, and a relatively inexpensive multi-core workstation offers substantially more 3 Hence, the challenge is transposed from the level of the national lab to the level of individual users, and from feasibility to practical ease of use. However, the impetus is no less real to reduce computation time from hours to the minutes required for interactive numerical explorations, and such an improvement would undoubtedly lead to further advances in our understanding of detonation phenomena.

Meanwhile, in parallel development, there has been considerable activity, centered around the Evans function [1, 39, 25], in the numerical evaluation of stability of viscous shock waves and other traveling front or pulse and boundary layer solutions arising in a variety of equations [14, 15, 16, 13, 28, 4, 26, 27, 5, 7, 8, 18, 9, some of which problems, see, e.g., 27, 5, 7, 18, exhibit complexity rivalling that of detonations. The authors and collaborators have developed a general model-independent method and set of numerical principles for the treatment of such problems [28, 54, encoded in the MATLAB-based platform STABLAB [ [6], which performs extremely well on all of the above-described applications.

At the same time, there has been a successful push to place detonation stability in a common framework with stability of shock waves [50, 35, 36, 29, 49, 52, 55. In particular, in 50, 29, 49, 52, 55, the determination of stability of both viscous (reactive Navier-Stokes) and inviscid (reactive Euler or ZND) detonations has been reduced to the computation of an Evans function defined exactly as in the viscous shock and other cases described above. Thus, it is a natural step to study ZND stability within this common framework, using the general tools of [28, 54 .

\footnotetext{
${ }^{2}$ A Cray X-MP $/ 48$ cost roughly $\$ 15-20 \mathrm{M}$ dollars in the mid-1980s, having 2 processors with a 105 $\mathrm{MHz}$ clock speed and a theoretical peak performance about 200 MFLOPS per processor or 400 MFLOPS total.

${ }^{3}$ A 2010 Mac Pro 8-core (2 quad-core Xeon processors) for example is a $\$ 4-5 \mathrm{k}$ system with a $2.5 \mathrm{GHz}$ clock speed and a theoretical peak performance around 10 GFLOPS per core or 80 GFLOPS total. Hence, it has roughly 200 times the processing power at a five thousandth the price (not even adjusting for inflation).
} 
In this paper, we do exactly that, proposing a new algorithm for the numerical determination of stability of ZND detonations derived from the point of view of [28, 54]. Surprisingly, though both are shooting methods, this is quite different from the Lee-Stewart algorithm currently in standard use, shooting from $x=-\infty$ to $x=0$ rather than from $x=0$ to $x=-\infty$ as in [34; indeed, it is more closely related to the original algorithm of Erpenbeck [21. The precise relations between the various methods are described in Section 4

The advantage of shooting from $-\infty$ to 0 is that we seek generalized eigenfunctions decaying exponentially at $-\infty$. Thus, in the forward direction $(-\infty \rightarrow 0)$, the desired solution grows exponentially, while error modes are exponentially damped. By contrast, integrating in the backward direction $(0 \rightarrow-\infty)$, the desired solution decays exponentially while error modes are exponentially amplified, a numerically undesirable situation ("numerical pitfall 1" of [54]). For this reason, we expect that our algorithm should be faster and better conditioned than the Lee-Stewart algorithm currently in use. However, there are other aspects that cloud the issue, in particular the singular perturbation structure that arises in the high-activation energy or "square-wave" limit in which instabilities are often studied [20, 22, 23, 17, 2, For this reason, a careful comparison of methods in physically relevant regimes is an important step before making conclusions.

In the present paper, we introduce the algorithm, and give some supporting numerical experiments for a simple model equation indicating the advantages of our approach. Followup work in [10, 11] indicates that, also in physically realistic settings, the algorithm performs favorably compared to the current standard. Specifically, the standard adaptive-mesh version of the algorithm described here appears to outperform the fixedmesh algorithm described in [34, 45] by 2-3 orders of magnitude. Much of this improvement appears to be due to the difference between fixed and adaptive mesh. However, even compared to an adaptive-mesh version of the method of Lee and Stewart, our algorithm appears to be 1-10 times faster, depending on the parameter regime: at the least, it is equivalent, and in some situations substantially more efficient.

Plan of the paper. In Section 2 we review the ZND equations and detonation structure. In Section 3, we give a simple derivation of the Evans/Lopatinski function condition for detonation stability from a general point of view following [50, 29]. For clarity, we specialize in most of the discussion to the single-species, ideal gas case with Arrhenius ignition dynamics, working in the same framework as in [34]. The general case is discussed briefly in Remark 5.1. In Section 4, we determine the relation between the derived Evans/Lopatinski condition, the related stability determinants of Erpenbeck [20, and Lee-Stewart [34]. In Section 5, we describe a proposed numerical implementation within the standard STABLAB package developed by the authors and collaborators. Finally, in Section 6, we present numerical experiments for a simple model indicating the advantages of integrating in the forward direction and factoring out expected decay at $-\infty$ as prescribed in $[28,54$. 


\section{ZND detonations.}

2.1. The model. In Eulerian coordinates the Zeldovich-von Neumann-Döring (ZND) equations of reacting gas dynamics in one space dimension may be written as

$$
\begin{aligned}
\rho_{t}+(\rho u)_{x} & =0, \\
(\rho u)_{t}+\left(\rho u^{2}+p\right)_{x} & =0, \\
(\rho \tilde{E})_{t}+((\rho \tilde{E}+p) u)_{x} & =0, \\
(\rho Y)_{t}+(\rho u Y)_{x} & =-\rho \varphi(T) K Y,
\end{aligned}
$$

where $\rho, u, p, \tilde{E}, T \in \mathbb{R}^{1}$ represent density, velocity, pressure, total energy, and temperature, and $Y=\left(Y_{1}, \ldots, Y_{r}\right) \in \mathbb{R}^{r}$ the mass fractions of reactants 4 Here, $\tilde{E}=u^{2} / 2+\tilde{e}$ is the nonreacting gas-dynamical energy $E=u^{2} / 2+e$ modified by chemical potential according to

$$
\tilde{e}=e+q Y,
$$

where $e$ is the specific internal energy of the gas and $q Y$ is the specific chemical energy. The matrix $K \in \mathbb{R}^{r \times r}$ and vector $q \in \mathbb{R}^{1 \times r}$ measure the rates of reaction and the heat released in the reaction, respectively, and $\varphi$ is an "ignition function" that is positive for $T$ above some ignition temperature $T_{i}$ and zero for $T \leq T_{i}$, serving to "turn on" the reaction. The matrix $-K$ is assumed to be stable, i.e., to have spectrum of strictly negative real part, so that the reaction in a quiescent flow indeed proceeds to the completely burned state $Y=0$. In the simplest case of a single-species, exothermic reaction, $Y \in \mathbb{R}^{1}$ is a scalar, and $K$ and $q$ are positive constants.

The system is closed by specifying the equations of state (i.e., thermodynamic relations) $p=p(\rho, e, Y)$ and $T=T(\rho, e, Y)$ and the ignition function. Standard assumptions (in particular, the ones made in [34, etc.) are the ideal gas laws

$$
p(\rho, e)=\Gamma \rho e, \quad T(e)=e / C_{v},
$$

where $\Gamma, C_{v}>0$ are constants determined by the nature of the gas, and the modified Arrhenius law

$$
\varphi(T)=\exp \left(-\frac{E_{A}}{R T}\right) \beta(T)
$$

where $E_{A}$ is the activation energy, $R=\gamma C_{V}$ is the gas constant, and $\beta$ is an artificial smooth cutoff function with the property that $\beta \equiv 1$ for $T \geq T^{i}$ and $\beta \equiv 0$ for $T \leq T_{i}$ 原 Under the usual assumptions, the specific form of the function $\beta$ plays no role in the analysis; see Remark 2.2 .

REMARK 2.1. More realistic rate laws $r(\rho, T, Y)$ may be considered in place of the linear law $r=-\rho \varphi(T) K Y$ with little additional difficulty [34; however, we lose the explicit form of the reaction profile (5.3) computed in Section 5.1 In the single-species case, these are equivalent.

\footnotetext{
${ }^{4}$ Alternatively, the equations may be written in terms of progress variables $\lambda_{j}=1-Y_{j}$ [22, 34, 36].

${ }^{5}$ The latter, standard modification circumvents the "cold-boundary difficulty" that the unburned state $Y \equiv 1$ is not an equilibrium for the exact Arrhenius law $\beta \equiv 1$, and so steady traveling detonation waves do not exist. Though not mentioned, this assumption is also made implicitly in [34, etc.
} 
2.2. Alternative formulation. Subtracting $q$ times the fourth equation of (2.1) from the third equation, we obtain the alternative formulation

$$
\begin{aligned}
\rho_{t}+(\rho u)_{x} & =0, \\
(\rho u)_{t}+\left(\rho u^{2}+p\right)_{x} & =0, \\
(\rho E)_{t}+((\rho E+p) u)_{x} & =\rho q \varphi(T) K Y, \\
(\rho Y)_{t}+(\rho u Y)_{x} & =-\rho \varphi(T) K Y
\end{aligned}
$$

in terms of the usual gas-dynamical variables $\rho, u, E$. We alternate between the two formulations as convenient for the analysis.

2.3. Detonation waves. For temperatures $T \leq T_{i}$ below ignition level, equations (2.1) evidently reduce to the usual Euler equations of nonreactive gas dynamics, with the reactants $Y$ convected passively by the velocity field $u$. In particular, so long as $T\left(\rho_{ \pm}, e_{ \pm}, Y_{0}\right)$ $\leq T_{i}$, they support as traveling-wave solutions ordinary gas-dynamical shock waves

$$
(\rho, u, E, Y)(x-s t)= \begin{cases}\left(\rho_{+}, u_{+}, E_{+}, Y_{0}\right), & x-s t>0 \\ \left(\rho_{-}, u_{-}, E_{-}, Y_{0}\right), & x-s t \leq 0\end{cases}
$$

satisfying the Rankine-Hugoniot conditions

$$
s[\rho]=[\rho u], \quad s[\rho u]=\left[\rho u^{2}+p\right], \quad s[\rho E]=[(\rho E+p) u], \quad[Y]=0,
$$

or, equivalently,

$$
s[\rho]=[\rho u], \quad s[\rho u]=\left[\rho u^{2}+p\right], \quad s[\rho \tilde{E}]=[(\rho \tilde{E}+p) u], \quad[Y]=0,
$$

where for an arbitrary function $h(\rho, u, \tilde{E}, Y),[h]:=h\left(\rho_{+}, u_{+}, \tilde{E}_{+}, Y_{+}\right)-h\left(\rho_{-}, u_{-}, \tilde{E}_{-}, Y_{-}\right)$ denotes the jump across the discontinuity. This also holds if there is no reactant, $Y_{0}=$ $(0, \ldots, 0)$.

If, on the other hand, $Y_{0} \neq(0, \ldots, 0)$, and $T_{+} \leq T_{i}$ but $T_{-} \geq T_{i}$, with $u_{ \pm}<s$ (alternatively, $T_{+} \geq T_{i}$ and $T_{-} \leq T_{i}$, with $u_{ \pm}>s$ ), then there appears a different type of traveling-wave solution known as a strong detonation, given by $(z=x-s t)$

$$
(\rho, u, E, Y)(z)= \begin{cases}\left(\rho_{+}, u_{+}, E_{+}, Y_{0}\right), & z>0 \\ (\bar{\rho}, \bar{u}, \bar{E}, \bar{Y})(z), & z \leq 0\end{cases}
$$

where $\bar{Y}(z)$ satisfies the smooth traveling-profile ODE

$$
(\bar{\rho}(\bar{u}-s) \bar{Y})^{\prime}=-\bar{\rho} \varphi(\bar{T}) K \bar{Y}
$$

on $(-\infty, 0]$, with initial condition $\bar{Y}(0)=Y_{0}$, decaying to the completely burned state $(0, \ldots, 0)$ as $z \rightarrow-\infty$, with $(\bar{\rho}, \bar{u}, \bar{E})=(\bar{\rho}, \bar{u}, \bar{E})(\bar{Y})$ determined through the generalized Rankine-Hugoniot relations

$$
\begin{aligned}
s \bar{\rho}-\bar{\rho} \bar{u} & =(s \rho-\rho u)_{ \pm}, \\
s \bar{\rho} \bar{u}-\left(\bar{\rho} \bar{u}^{2}+\bar{p}\right) & =\left(s \rho u-\left(\rho u^{2}+p\right)\right)_{ \pm}, \\
s \bar{\rho} \overline{\tilde{E}}-(\bar{\rho} \overline{\tilde{E}}+\bar{p}) \bar{u} & =(s \rho \tilde{E}-(\rho \tilde{E}+p) u)_{ \pm}
\end{aligned}
$$


obtained by integrating the remaining traveling-profile equations

$$
\begin{aligned}
(s \bar{\rho}-\bar{\rho} \bar{u})^{\prime} & =0, \\
\left(s \bar{\rho} \bar{u}-\left(\bar{\rho} \bar{u}^{2}+\bar{p}\right)\right)^{\prime} & =0, \\
(s \bar{\rho} \overline{\tilde{E}}-(\bar{\rho} \overline{\tilde{E}}+\bar{p}) \bar{u})^{\prime} & =0
\end{aligned}
$$

from 0 to $z$ (where $z<0$ ) and recalling the Rankine-Hugoniot conditions (2.6) satisfied across the jump at $z=0$.

That is, strong detonations moving to the right with respect to fluid velocity $u$ (i.e., $u<s$, where $s$ is the speed of the detonation) have the structure of an initiating gasdynamical shock called the Neumann shock, which rapidly compresses the gas, raising the temperature to the point of ignition, followed by a reaction zone (the profile $(\bar{\rho}, \bar{u}, \bar{E}, \bar{Y})$ ) resolving to the final burned state. This characteristic "detonation spike" in temperature and pressure profiles agrees well with observed features in laboratory experiments.

Substituting into (2.8) the first relation in (2.9) and introducing the constant $m:=$ $(\rho(s-u))_{ \pm}$, we obtain the simplified reaction equation

$$
Y^{\prime}=m^{-1} \rho \varphi(T) K Y
$$

that we will actually use to solve for the profile. Further simplifying (2.9), we obtain

$$
\begin{aligned}
s \bar{\rho}-\bar{\rho} \bar{u} & =(s \rho-\rho u)_{ \pm}, \\
s \bar{\rho} \bar{u}-\left(\bar{\rho} \bar{u}^{2}+\bar{p}\right) & =\left(s \rho u-\left(\rho u^{2}+p\right)\right)_{ \pm}, \\
s \bar{\rho} \bar{E}-(\bar{\rho} \bar{E}+\bar{p}) \bar{u}+m q \bar{Y} & =(s \rho E-(\rho E+p) u+m q Y)_{ \pm} .
\end{aligned}
$$

An application of the Implicit Function Theorem reveals that (2.9) (as, likewise, the original ODE (2.10) ) may be solved for $(\bar{\rho}, \bar{u}, \bar{E})$ in terms of $\bar{Y}$ so long as the gas-dynamical state $(\bar{\rho}, \bar{u}, \bar{E})$ remains noncharacteristic with respect to speed $s$, or, equivalently, the Rankine-Hugoniot relation (2.12) remains full rank in $(\bar{\rho}, \bar{u}, \bar{E})$. For typical reactions and equations of state, in particular ideal gas dynamics with single exothermic reaction, this condition holds for all solutions of (2.12) with $\bar{Y}_{j} \geq 0$, except for special limiting values of $s$ for which the asymptotic state $\bar{Y}=0$ is characteristic, or "sonic"; see, e.g., [35]. These limiting, characteristic waves are called Chapman-Jouget detonations and have a special place in the theory. The usual, noncharacteristic type are called overdriven detonations.

For our present purposes, the main import of characteristicity is that the eigenvalue equation becomes singular at $x \rightarrow-\infty$ in the coordinates we use here, complicating the discussion. For simplicity, we restrict hereafter to the overdriven case. The ChapmanJouget case may be treated similarly using ideas of [34; see Remark 5.1.

REMARK 2.2. For the modified Arrhenius ignition function (2.3), a standard assumption is that $\bar{T} \geq T^{i}$, all $x \leq 0, T_{+} \leq T_{i}$, so that $\beta \equiv 1$ for $x \leq 0$ and $\beta \equiv 0$ for $x \geq 0$. Under this assumption, the specific form of the cutoff $\beta$ plays no role in the analysis.

3. Linearized stability analysis: the Evans-Lopatinski determinant. We now carry out a linearized interface analysis, loosely following [29] Setting $V:=(\rho, u, e)^{T}$,

\footnotetext{
${ }^{6}$ See also the related [50, 35, 36], and the original treatments in [19, 20, 34, etc.
} 
write (2.4) in abstract form as

$$
F^{0}(W)_{t}+F^{1}(W)_{x}=R(W),
$$

$W, F^{j}, R \in \mathbb{R}^{3+r}$, where

$$
\begin{gathered}
W:=\left(\begin{array}{c}
V \\
Y
\end{array}\right), \quad F^{j}:=\left(\begin{array}{c}
f^{j}(W) \\
Y g^{j}(V)
\end{array}\right), \quad R:=\left(\begin{array}{c}
Q K Y \psi(W) \\
-K Y \psi(W)
\end{array}\right), \\
f^{0}:=\left(\begin{array}{c}
\rho \\
\rho u \\
\rho\left(e+u^{2} / 2\right)
\end{array}\right), \quad f^{1}:=\left(\begin{array}{c}
\rho u \\
\rho u^{2}+p(\rho, e, Y) \\
\left(\rho\left(e+u^{2} / 2\right)+p(\rho, e, Y)\right) u
\end{array}\right), \\
g^{0}:=\rho, \quad g^{1}=\rho u, \quad Q:=\left(\begin{array}{ccc}
0 & \cdots & 0 \\
q_{1} & \cdots & q_{r}
\end{array}\right), \quad \psi:=\rho \phi(T(\rho, e, Y)),
\end{gathered}
$$

with $V, f^{j} \in \mathbb{R}^{3}, Y \in \mathbb{R}^{r}, g^{j}, \psi \in \mathbb{R}^{1}, Q \in \mathbb{R}^{3 \times r}$.

REmark 3.1. A minor departure from [19, 20, 50, 29] is to admit the possible dependence of pressure and temperature on the chemical makeup of the gas $(Y)$, an important feature in realistic modeling of reactive flow.

To investigate solutions in the vicinity of a discontinuous detonation profile, we postulate existence of a single shock discontinuity at location $X(t)$, and reduce to a fixedboundary problem by the change of variables $x \rightarrow x-X(t)$. In the new coordinates, the problem becomes

$$
F^{0}(W)_{t}+\left(F^{1}(W)-X^{\prime}(t) F^{0}(W)\right)_{x}=R(W), \quad x \neq 0,
$$

with jump condition

$$
X^{\prime}(t)\left[F^{0}(W)\right]-\left[F^{1}(W)\right]=0,
$$

$[h(x, t)]:=h\left(0^{+}, t\right)-h\left(0^{-}, t\right)$ as usual denoting jump across the discontinuity at $x=0$.

3.1. Linearization. Without loss of generality, suppose for simplicity that the background profile $\bar{W}$ is a steady detonation, i.e., $s=0$; hence $(\bar{W}, \bar{X})=(\bar{W}, 0)$ is also a steady solution of (3.4)-(3.5). Linearizing (3.4)-(3.5) about the solution $(\bar{W}, 0)$, we obtain the linearized equations

$$
\begin{gathered}
A^{0}\left(W_{t}-X^{\prime}(t) \bar{W}^{\prime}(x)\right)+\left(A^{1} W\right)_{x}=C W, \\
X^{\prime}(t)\left[F^{0}(\bar{W})\right]-\left[A^{1} W\right]=0, \quad x=0, \\
A^{j}:=(\partial / \partial W) F^{j}, \quad C:=(\partial / \partial W) R .
\end{gathered}
$$

3.2. Reduction to homogeneous form. As pointed out in [29], it is convenient for the stability analysis to eliminate the front from the interior equation (3.6). Therefore, we reverse the original transformation to linear order by the change of dependent variables

$$
W \rightarrow W-X(t) \bar{W}^{\prime}(x),
$$

following the calculation

$$
W(x-X(t), t)-W(x, t) \sim-X(t) W_{x}(x, t) \sim-X(t) \bar{W}^{\prime}(x),
$$

approximating to linear order the original, nonlinear transformation. Substituting (3.9) in (3.6)-(3.7), and noting that $x$-differentiation of the steady profile equation $F^{1}(\bar{W})_{x}=$ 
$R(\bar{W})$ gives

$$
\left(A^{1}(\bar{W}) \bar{W}^{\prime}(x)\right)_{x}=C(\bar{W}) \bar{W}^{\prime}(x),
$$

we obtain modified, homogeneous interior equations

$$
A^{0} W_{t}+\left(A^{1} W\right)_{x}=C W
$$

agreeing with those that would be obtained by a naive calculation without consideration of the front, together with the modified jump condition

$$
X^{\prime}(t)\left[F^{0}(\bar{W})\right]-X(t)\left[A^{1} \bar{W}^{\prime}(x)\right]-\left[A^{1} W\right]=0
$$

correctly accounting for front dynamics.

The reduction to homogeneous interior equations puts the linearized problem in a standard linear boundary-value-problem format for which stability may be investigated in a straightforward fashion by the construction of an Evans/Lopatinski determinant. Besides simplifying considerably Erpenbeck's original derivation of his equivalent stability function 20, the homogeneous format makes possible the application of standard numerical Evans function techniques for its evaluation. This useful reduction was first carried out, in slightly different form, in [29. The transformation (3.9) is of general use in interface problems, comprising the "good unknown" of Alinhac [3]. A similar discussion in the simpler context of shock waves may be found in 24]; however, in this case, $\bar{W}^{\prime}(x) \equiv 0$, and so the transformation (3.9) does not make itself evident, nor do front dynamics modify (3.12).

3.3. The stability determinant. Seeking normal mode solutions $W(x, t)=e^{\lambda t} W(x)$, $X(t)=e^{\lambda t} X, W$ bounded, of the linearized equations (3.11)-(3.12), we are led to the generalized eigenvalue equations

$$
\begin{gathered}
\left(A^{1} W\right)^{\prime}=\left(-\lambda A^{0}+C\right) W, \quad x \neq 0, \\
X\left(\lambda\left[F^{0}(\bar{W})\right]-\left[A^{1} \bar{W}^{\prime}(x)\right]\right)-\left[A^{1} W\right]=0,
\end{gathered}
$$

where "" denotes $d / d x$, or, setting $Z:=A^{1} W$, to

$$
\begin{gathered}
Z^{\prime}=G Z, \quad x \neq 0, \\
X\left(\lambda\left[F^{0}(\bar{W})\right]-\left[A^{1} \bar{W}^{\prime}(x)\right]\right)-[Z]=0,
\end{gathered}
$$

with

$$
G:=\left(-\lambda A^{0}+C\right)\left(A^{1}\right)^{-1} .
$$

Here, we are implicitly using the following elementary observation.

Lemma 3.2. $A^{1}(\bar{W}(x))$ is invertible for all $x$ such that $\partial f / \partial V$ is invertible (i.e. $V$ is noncharacteristic as a gas-dynamical state with $Y$ held fixed).

Proof. Similarly as in the discussion of existence of steady profiles, we may by subtracting $Y$ times the first row of $A^{1}$ from the block $Y$-row, reduce $A^{1}$ to block upper-triangular form, with diagonal blocks $\partial f / \partial V$ and $g^{1}(V, Y) I_{r \times r}$ with $g^{1}(V, Y)=\rho u \neq 0$.

REMARK 3.3. As discussed in Section 2.1, this assumption is essentially necessary already for existence of a steady profile. In particular, it is satisfied for the usual ideal gas equation of state.

We require also the following fundamental properties. 
Lemma 3.4 (19, 20, 29]). On $\Re e \lambda>0$, the limiting $(3+r) \times(3+r)$ coefficient matrices $G_{ \pm}:=\lim _{z \rightarrow \pm \infty} G(z)$ have unstable subspaces of fixed rank: full rank $3+r$ for $G_{+}$and rank $2+r$ for $G_{-}$. Moreover, these subspaces have continuous limits as $\Re e \lambda \rightarrow 0$.

Proof. This is a straightforward calculation using the fact that $G_{ \pm}$are block uppertriangular in $(V, Y)$; see, e.g., [19, 20, 50, 29] in the case that $f, g$ depend only on $V$.

Corollary 3.5 ([50, 29]). On $\Re e \lambda>0$, the only bounded solution of (3.13) for $x>0$ is the trivial solution $W \equiv 0$. For $x<0$, the bounded solutions consist of an $(r+$ 2)-dimensional subspace $\operatorname{Span}\left\{Z_{1}^{-}, \ldots, Z_{r+2}^{-}\right\}(\lambda, x)$ of exponentially decaying solutions, analytic in $\lambda$ and tangent as $x \rightarrow-\infty$ to the subspace of exponentially decaying solutions of the limiting, constant-coefficient equations $Z^{\prime}=G_{-} Z$; moreover, this subspace has a continuous limit as $\Re e \lambda \rightarrow 0$.

Proof. The first observation is immediate, using the fact that $G$ is constant for $x>$ 0 . The second follows from asymptotic ODE theory, using the "gap" or "conjugation" lemmas of [25, 30, 38, together with the fact that $G$ decays exponentially to its end state as $x \rightarrow-\infty$. See [29, 52, 55] for details.

Definition 3.6. We define the Evans-Lopatinski determinant

$$
\begin{aligned}
& D(\lambda):=\operatorname{det}\left(Z_{1}^{-}(\lambda, 0), \quad \ldots, \quad Z_{r+2}^{-}(\lambda, 0), \quad \lambda\left[F^{0}(\bar{W})\right]-\left[A^{1} \bar{W}^{\prime}(x)\right]\right) \\
& =\operatorname{det}\left(Z_{1}^{-}(\lambda, 0), \quad \ldots, \quad Z_{r+2}^{-}(\lambda, 0), \quad \lambda\left[F^{0}(\bar{W})\right]+A^{1} \bar{W}^{\prime}\left(0^{-}\right)\right),
\end{aligned}
$$

where $Z_{j}^{-}(\lambda, x)$ are as in Corollary 3.5 ,

The function $D$ is exactly the stability function derived in a different form by Erpenbeck [20; see Section 4.2 below. The formulation (3.16) is of the standard form arising in the simpler context of (nonreactive) shock stability [37, 19]. Evidently (by (3.14) combined with Corollary (3.5), $\lambda$ is a generalized eigenvalue/normal mode for $\Re e \lambda \geq 0$ if and only if $D(\lambda)=0$.

REMARK 3.7. As noted in [52, 55], consideration of the traveling-wave equation $F(W)^{\prime}=A W^{\prime}=R(W)$ yields the simpler formula

$$
D(\lambda)=\operatorname{det}\left(Z_{1}^{-}(\lambda, 0), \quad \ldots, \quad Z_{r+2}^{-}(\lambda, 0), \quad \lambda\left[F^{0}(\bar{W})\right]+R\left(\bar{W}\left(0^{-}\right)\right)\right) .
$$

3.4. Dual formulation. The $(n+r) \times(n+r)$ determinant (3.17) may be expressed more succinctly in dual form

$$
D(\lambda)=\tilde{Z}^{-}(\lambda, 0) \cdot\left(\lambda\left[F^{0}(\bar{W})\right]+R\left(\bar{W}\left(0^{-}\right)\right)\right),
$$

where $\tilde{Z}^{-}(\lambda, x)$ is the cross product $Z_{1}^{-} \wedge \cdots \wedge Z_{r+2}^{-}(\lambda, x)$ defined by

$$
\tilde{Z}^{-} \cdot x=\operatorname{det}\left(Z_{1}^{-}, \quad \ldots, \quad Z_{r+2}^{-}, \quad x\right) .
$$

The vector $\tilde{Z}^{-}$may alternatively be characterized directly as the unique up to constant factor bounded solution on $x \leq 0$ of the adjoint ODE

$$
\tilde{Z}^{\prime}=-G^{*} \tilde{Z}
$$

which, as $x \rightarrow-\infty$ is both exponentially decaying and tangent to the corresponding exponentially decaying one-dimensional subspace of bounded solutions of the limiting 
constant-coefficient equations $\tilde{Z}^{\prime}=-G_{-}^{*} \tilde{Z}$. It may be specified analytically in $\lambda$ by the additional requirement

$$
\tilde{\Pi}\left(\tilde{Z}^{-}\right)^{\operatorname{conj}}(-M)=\ell(\lambda)
$$

$M>0$, where $\ell$ is an analytically chosen left eigenvector of $G_{-}(\lambda)$ associated with the unique eigenvalue $g_{-}(\lambda)$ of negative real part and $\tilde{\Pi}$ the associated eigenprojection. Here, and elsewhere, ${ }^{c o n j}$ denotes complex conjugate. By (3.20) together with the tangency property, $\tilde{Z}^{-}$is well-approximated at $x=-M$, for $M>0$ sufficiently large, by

$$
\tilde{Z}^{-}(-M)=\ell^{\operatorname{conj}}(\lambda) \text {. }
$$

This reduces the approximate evaluation of $D(\cdot)$ to the straightforward and extremely well-conditioned numerical problem of integrating a single exponentially growing (in the forward direction) mode from $x=-M$ to $x=0$. The stability of the computation derives from the fact that errors lying in other, exponentially decaying modes, are exponentially damped [54].

Alternate initialization. Alternatively, following [14, 15, 16, 13, $\tilde{Z}^{-}$may be specified by boundary conditions at $-\infty$, via

$$
\lim _{x \rightarrow-\infty} e^{g_{-}^{c o n j} x} \tilde{Z}^{-}(x)=\ell^{c o n j}(\lambda),
$$

whence 3.21 becomes

$$
\tilde{Z}^{-}(-M)=e^{-g_{-}^{c o n j} M} \ell^{c o n j}(\lambda) .
$$

This is the method that we prescribe here. It has the advantage of removing the dependence of $\tilde{Z}^{-}$on the artificial parameter $M$, allowing the flexible choice of $M$ in different parameter regimes, as dictated by numerical considerations, while preserving analyticity. However, in practice, there is usually not much difference between (3.21) and (3.23). In particular, if, as in [34], one is not interested in analyticity, then one may vary $M$ freely in (3.21) as well.

\section{Relations to other methods.}

4.1. Relation to the method of Lee and Stewart. Denoting by $Z_{0}$ the solution on $x \leq$ 0 of the forward eigenvalue ODE (3.13) with initial conditions $Z_{0}(0):=\lambda\left[F^{0}(\bar{W})\right]+$ $R\left(W\left(0^{-}\right)\right)$, we have by standard duality properties that

$$
\tilde{Z}^{-} \cdot Z_{0}(\lambda, x) \equiv D(\lambda)
$$

is independent of $x \leq 0$, or $\left(\tilde{Z}^{-} \cdot Z_{0}\right)^{\prime}(\lambda, x) \equiv 0$. Taking $x=-M$ and recalling (3.21), we arrive at the alternative Evans-Lopatinski approximation

$$
D(\lambda) \sim \ell^{\operatorname{conj}}(\lambda) \cdot Z_{0}(\lambda,-M)
$$

used by Lee and Stewart 34, where $\ell^{\operatorname{conj}} \cdot Z_{0}(-M)=0$ is their "nonradiative condition" enforcing boundedness of $Z_{0}$. The solution of $Z_{0}$ from $x=0$ to $x=-M$, on the other hand, is numerically comparatively ill-conditioned in the vicinity of roots of $D(\cdot)$, since $Z_{0}$ in this regime is approximately exponentially decaying in the backward direction 
while errors are exponentially growing 7 The version (3.18) is therefore much preferable from the numerical point of view, at least when used (as here, and in [34) as a shooting method.

4.2. Relation to the method of Erpenbeck. Erpenbeck 21 computes $\tilde{Z}^{-}$in much the same way as we do here. However, in place of the homogeneous duality relation (4.1), he uses the "inhomogeneous Abel relation"

$$
\left.\left(\tilde{Z}^{-} \cdot \hat{Z}_{0}\right)^{\prime}(\lambda, x)\right)=\tilde{Z} \cdot \lambda \bar{W}^{\prime}(x),
$$

valid for the solution $\hat{Z}_{0}$ of the inhomogeneous equation $Z^{\prime}=G Z+\lambda \bar{W}^{\prime}(x)$ with initial data $\hat{Z}_{0}(0):=\lambda[\bar{W}]$ deriving from the unmodified equations (3.6)-(3.7), together with $\bar{W}^{\prime}(-\infty)=0$, to evaluate

$$
D(\lambda)=\int_{-\infty}^{0} \tilde{Z}^{-}(y) \cdot \lambda \bar{W}^{\prime}(y) d y+\tilde{Z}^{-}(0) \cdot \lambda[\bar{W}]
$$

Though it is mathematically equivalent to the homogeneous scheme described above, this has the disadvantage that it is difficult to implement adaptive control on truncation error simultaneously for the ODE and quadrature steps. Indeed, the method is in general a bit more cumbersome to implement and understand than either of the previous two described methods. As a one-time cost, the latter is a rather minor point. However, the implications of the former for performance appear to be significant. Our experience in similar Evans function-type shooting computations [16, 28, 4, 26] of spectra of asymptotically constant-coefficient operators is that a fixed-step scheme can be orders of magnitude slower than a comparable adaptive scheme; see [54] for a general discussion of performance of numerical Evans/Lopatinski solvers. Moreover, even in the solution of $\tilde{Z}$ alone, the use of an adaptive solver without factoring out expected decay is much less effective in our experience ("numerical pitfall 3" of [54]).

4.3. Expression as boundary-value solver. We mention in passing an alternative "local Evans function" formulation in the spirit of [34], suggested by Sandstede 41] as a general method for numerical Evans function investigations using collocation/continuation rather than shooting. By the analysis of the previous subsections, we may recast the eigenvalue equation (3.13)-(3.14) as in [34] as an overdetermined two-point boundary-value problem $Z^{\prime}=G Z$ with $r+4$ boundary conditions

$$
Z(0):=\lambda\left[F^{0}(\bar{W})\right]+R\left(\bar{W}\left(0^{-}\right)\right), \quad \lim _{x \rightarrow-\infty} \ell^{\text {conj }} \cdot Z(x)=0 .
$$

Relaxing at random one of the $r+3$ conditions at $x=0$, say the requirement on the $j$ th coordinate, we generically obtain a well-posed boundary-value problem with the correct number $r+3$ of boundary conditions; one of the coordinates will always suffice. More, the projective boundary-condition at $x=-\infty$ is numerically "correct", making this problem extremely well-conditioned for solution by collocation/continuation methods (see, e.g., [40]). Defining $Z(\lambda, x)$ to be the solution of this relaxed problem, we may then define a

\footnotetext{
${ }^{7}$ More precisely, they solve the inhomogeneous equations $Z_{0}^{\prime}=G Z_{0}+\lambda \bar{W}^{\prime}(x)$ with initial data $\hat{Z}_{0}(0):=\lambda[\bar{W}]$, and compute $\ell^{c o n j}(\lambda) \cdot Z_{0}(\lambda,-M) \sim \ell^{c o n j}(\lambda) \cdot \hat{Z}_{0}(\lambda,-M)$, which is numerically equivalent. Here we are using $\hat{Z}_{0}-Z_{0}=\bar{W}^{\prime}(x) \rightarrow 0$ as $x \rightarrow-\infty$.
} 
local, analytic Evans function

$$
\tilde{D}(\lambda):=e_{j} \cdot\left(Z(\lambda, 0)-\left(\lambda\left[F^{0}(\bar{W})\right]+R\left(\bar{W}\left(0^{-}\right)\right)\right)\right)
$$

that is numerically well-conditioned and vanishes if and only if $\lambda$ is an eigenvalue. This gives a second way to convert (4.4) into a numerically well-conditioned problem, though the speed and simplicity of shooting is lost in this approach, along with global analyticity useful for winding number calculations. We shall not investigate this method here, but note that it could be useful in extreme conditions such as the ultra-high activation energy limit 17 .

5. Numerical implementation. We now describe in detail the numerical algorithm proposed to compute (3.18), following the general approach set out in [16, 28, 53, 54.

5.1. Computing the profile. In Evans function computations, a delicate aspect is often the computation of the background nonlinear profile. We sidestep this issue by the explicit solution technique used in [19, 20, 34, modified slightly to accommodate the multi-species case (specifically, the simplified uniform ignition case considered here).

Introducing the new variable $y$ defined by

$$
d y / d x=m^{-1} \rho \varphi(T), \quad y(0)=0,
$$

where $m:=(\rho(s-u))_{ \pm}$, we reduce the reaction equation (2.11) to

$$
d Y / d y=K Y, \quad Y(0)=Y_{0},
$$

obtaining an explicit solution

$$
Y(y)=e^{K y} Y_{0}
$$

from which the full profile can be recovered through (2.12), either by explicit calculation, as carried out for ideal gas dynamics in Appendix B, or, more generally, by Newton iteration.

REMARK 5.1. In the single-species case, (5.2) reduces to the change of coordinates $x \rightarrow y:=\log Y$ used in [34; general, nonlinear rate laws, or Chapman-Jouget waves, may be accommodated by a change of variables $x \rightarrow Y^{r}$ for appropriate $r$, as discussed in 34 .

5.2. Computing the stability determinant. The linearized stability analysis can then be carried out in the variable $y$ defined in (5.1), using the instantaneous change of variables formula

$$
d x / d y=m /(\bar{\rho} \varphi(\bar{T})), \quad y \leq 0 .
$$

REMARK 5.2. Since the right-hand side of (5.4) is uniformly positive and bounded, the variables $x$ and $y$ are equivalent in the sense that $C x \leq y \leq x / C$ for $x, y \leq 0$, for some $C>0$.

Specifically, we solve from $y=-M$ to $y=0$ the $\operatorname{ODE}(d / d y) \tilde{Z}=-m /(\bar{\rho} \varphi(\bar{T})) G^{*}(y) \tilde{Z}$, with initial condition $\tilde{Z}^{-}(-M)=e^{-g_{-}^{\text {conj }}(\lambda) M} \ell^{\text {conj }}(\lambda), M>0$ sufficiently large, where the vector $\ell(\lambda)$ and limiting eigenvalue $g_{1}=\left(u_{-}+c_{-}\right)^{-1}$ are as computed in (A.2) and (A.3) of Appendix A, the coefficient $G(\lambda, \bar{V}, \bar{Y})$ is as described in (3.15), (3.8), and (3.2)(3.3), and the profile $(\bar{V}, \bar{Y})(y)$ is as computed in Appendix B As prescribed in (3.18), we may then compute the stability determinant $D(\lambda)=\tilde{Z}^{-}(\lambda, 0) \cdot\left(\lambda\left[F^{0}(\bar{W})\right]+R\left(\bar{W}^{\prime}\left(0^{-}\right)\right)\right)$. 
More precisely, we may solve the numerically more advantageous equations

$$
(d / d y) \hat{Z}=-(m / \bar{\rho} \varphi(\bar{T}))\left(G(y)+g_{-}(\lambda) I\right)^{*} \hat{Z},
$$

with initial conditions $\hat{Z}(y):=e^{-\left(m g_{-}^{\text {conj }} / \bar{\rho} \varphi(\bar{T}) y\right.} \tilde{Z}(y)$, and compute

$$
D(\lambda)=\hat{Z}^{-}(\lambda, 0) \cdot\left(\lambda\left[F^{0}(\bar{W})\right]+R\left(\bar{W}\left(0^{-}\right)\right)\right) .
$$

This may readily be computed with good results by an adaptive solver such as the standard RK45; see [16, 28, 54 for further discussion.

5.3. Determination of stability: winding number vs. stability curves. With an Evans solver in hand, stability may be checked either by winding number computations as in [21, 4, 26], or by root-following methods based on the Implicit Function Theorem, as in 34. In the first method, a large semicircle $S$ centered at the origin and lying in $\Re \lambda \geq 0$ is mapped by $D$, and the number of zeros of $D$ (unstable normal modes) lying within $S$ computed using the principle of the argument, making use of the underlying analyticity of $D$. Unstable modes lying outside $S$ may be excluded by a separate, asymptotic, argument based on the high-frequency behavior of $D$ [14, 15, 26]; for implementations in the context of ZND, see [55, 33] (analytical) or [10, 11] (numerical). In the second method, individual roots are followed, avoiding the need to compute around a contour, but typically requiring an extra Newton iteration with each change in model parameters; see, for example, 34, 44. Both are by now completely standard.

6. A simple model problem. We conclude by an examination of efficiency within the context of a simple but illustrative model problem. Consider the ODE

$$
y^{\prime}=A(x, \lambda) y, \quad A(x, \lambda)=\lambda\left(\begin{array}{cc}
\frac{1}{2} & 0 \\
\frac{1}{c} e^{2 x} & \frac{-1}{2}
\end{array}\right)
$$

defined on $-\infty<x \leq 0, x \in \mathbb{R}, \lambda \in \mathbb{C}, y \in \mathbb{C}^{2}$, with boundary conditions $y \sim e^{\lambda x / 2}(1,0)^{T}$ as $x \rightarrow-\infty$ and $y(0)=(1,0)^{T}$, modeling a variable-coefficient eigenvalue problem of the form arising in ZND, where the coefficient $c \neq 0$ encodes the rapidity of exponential decay. As for ZND, the coefficient matrix is exponentially asymptotically constant as $x \rightarrow-\infty$, with size growing linearly in $\lambda$, and has a unique decaying mode as $x \rightarrow-\infty$ for all $\Re \lambda>0$, extending continuously to $\Re \lambda=0$. Thus, we may expect somewhat similar behavior, at least away from the high-activation energy "square-wave" regime.

In this context, our proposed algorithm consists of factoring out the expected decay $e^{\lambda x / 2}$ from the solution to obtain a "neutral" equation

$$
\hat{y}^{\prime}=\hat{A}(x, \lambda) \hat{y}, \quad \hat{A}(x, \lambda)=\lambda\left(\begin{array}{cc}
0 & 0 \\
\frac{1}{c} e^{2 x} & -1
\end{array}\right),
$$

$y:=\hat{y} e^{\lambda x / 2}$, then solving (6.2) from $x=-M$ to $x=0$ and checking whether $\hat{y}(0)$ lies parallel to $(1,0)^{T}$. For reasonable values of $c$, a computational domain of $M=$ 5 is sufficient. The method of Lee and Stewart, consists roughly of integrating the original equation (6.1) from $x=0$ to $x=-M$; the method of Erpenbeck consists roughly of integrating (6.1) from $x=-M$ to $x=0$ without first factoring out the expected exponential decay. For comparison, we considered also a worst-case scenario with maximum amplification of error modes, integrating (6.2) from $x=0$ to $x=-\infty$. 
TABLE 1. Runs for Eq. (6.2). Forward corresponds to our proposed method, with expected decay factored out. Backward is a worst-case scenario not corresponding to any of the methods considered.

\begin{tabular}{|r|c|c|c|c|c|c|}
\hline & \multicolumn{6}{|c|}{ mesh points } \\
\hline & \multicolumn{2}{|c|}{ forward integration } & \multicolumn{3}{|c|}{ backward integration } \\
\hline$\lambda$ & $c=10$ & 100 & 1000 & $c=10$ & 100 & 1000 \\
\hline $1.0+0 \mathrm{i}$ & 19 & 14 & 12 & 26 & 24 & 19 \\
$4.0+0 \mathrm{i}$ & 43 & 29 & 19 & 94 & 92 & 88 \\
$16.0+0 \mathrm{i}$ & 107 & 76 & 51 & 363 & 361 & 357 \\
$64.0+0 \mathrm{i}$ & 261 & 191 & 138 & 1438 & 1436 & 1432 \\
$256.0+0 \mathrm{i}$ & 657 & 519 & 427 & 3177 & 3186 & 3192 \\
\hline $0.4+0 \mathrm{i}$ & 14 & 12 & 11 & 17 & 14 & 11 \\
$0.4+1 \mathrm{i}$ & 17 & 13 & 12 & 30 & 27 & 18 \\
$0.4+4 \mathrm{i}$ & 43 & 29 & 19 & 100 & 97 & 73 \\
$0.4+16 \mathrm{i}$ & 111 & 77 & 51 & 385 & 382 & 296 \\
$0.4+64 \mathrm{i}$ & 317 & 224 & 177 & 1528 & 1523 & 1185 \\
$0.4+256 \mathrm{i}$ & 1088 & 870 & 827 & 6104 & 6086 & 4738 \\
\hline
\end{tabular}

We computed all with the adaptive-mesh RK45 algorithm (ode45) supported in MATLAB 8 with error tolerance set at the standard level $10^{-5}$ used for Evans computations [26, 5, 7, 8, measuring efficiency by the number of mesh points/function calls required to complete the computation. Extreme cases are $\lambda$ real (the "best" case), with a spectral gap between exponentially growing and exponentially decaying modes at $-\infty$ and $\lambda$ imaginary (the "worst" case from our standpoint), with neither spectral gap nor exponential decay. From the standpoint of the Lee-Stewart method, the best and worst cases would appear to be reversed.

The results, displayed in Tables 1 and 2 for a typical value $c=10$, indicate that the proposed new algorithm performs 1-5 times faster than (adaptive versions of) either the Erpenbeck or Lee-Stewart methods, depending on the value of $\lambda$, with particular improvement as $|\lambda|$ becomes large. It should be noted, moreover, that this is only a comparison of speed (number of mesh points) for the various methods to produce output with fixed truncation error. If we consider also accuracy, i.e., convergence error, then the results could be expected to be more dramatic, since both Lee-Stewart and Erpenbeck methods are numerically less well-posed than the forward "neutral" algorithm that we propose.

Appendix A. Calculation of $\ell$. In this appendix, we show how to calculate for general equations of state the initializing vector $\ell(\lambda)$ used in (3.21), the unique stable

\footnotetext{
${ }^{8}$ In practice, faster than corresponding fixed-mesh methods [54, 10, 11.
} 
TABle 2. Runs for Eq. 6.1). Forward corresponds to the Erpenbeck method, backward to the Lee-Stewart method.

\begin{tabular}{|r|c|c|c|c|c|c|}
\hline & \multicolumn{6}{|c|}{ mesh points } \\
\hline & forward integration & \multicolumn{3}{l|}{ backward integration } \\
\hline$\lambda$ & $c=10$ & 100 & 1000 & $c=10$ & 100 & 1000 \\
$1.0+0 \mathrm{i}$ & 23 & 19 & 15 & 19 & 17 & 15 \\
$4.0+0 \mathrm{i}$ & 61 & 58 & 56 & 52 & 50 & 49 \\
$16.0+0 \mathrm{i}$ & 181 & 181 & 181 & 186 & 184 & 183 \\
$64.0+0 \mathrm{i}$ & 719 & 719 & 719 & 723 & 721 & 721 \\
$256.0+0 \mathrm{i}$ & 2868 & 2868 & 2868 & 2873 & 2871 & 2870 \\
\hline $0.4+0 \mathrm{i}$ & 16 & 13 & 12 & 17 & 13 & 12 \\
$0.4+1 \mathrm{i}$ & 20 & 17 & 15 & 20 & 17 & 15 \\
$0.4+4 \mathrm{i}$ & 55 & 52 & 50 & 54 & 52 & 50 \\
$0.4+16 \mathrm{i}$ & 196 & 194 & 193 & 197 & 195 & 193 \\
$0.4+64 \mathrm{i}$ & 765 & 765 & 765 & 775 & 771 & 765 \\
$0.4+256 \mathrm{i}$ & 3055 & 3055 & 3055 & 3084 & 3074 & 3055 \\
\hline \hline
\end{tabular}

left eigenvector of the limiting coefficient matrix

$$
G_{-}=\left(-\lambda A_{-}^{0}+C_{-}\right)\left(A_{-}^{1}\right)^{-1}=\left(\begin{array}{cc}
-\lambda f_{V-}^{0}\left(f_{V-}^{1}\right)^{-1} & \left(\lambda f_{V-}^{0}\left(f_{V-}^{1}\right)^{-1} f_{Y-}^{1}+Q K \psi_{-}\right)\left(g_{-}^{1}\right)^{-1} \\
0 & \left(-\lambda g_{-}^{0}-K \psi_{-}\right)\left(g_{-}^{1}\right)^{-1}
\end{array}\right),
$$

where for a general function $h(V, Y)$, we use $h_{-}$to denote $h\left(V_{-}, Y_{-}\right)$. Here, we have strongly used $Y_{-}=0$ to obtain the simple upper block-triangular form.

By the upper block-triangular form of $G_{-}$, and the fact that the lower right-hand block has spectrum of positive real part for $\Re e \lambda>0$ (since $g^{0}>0$ always, $g^{1}<0$ for right-moving detonations, and $-K$ is assumed to have spectrum of negative real part), we find that $\ell^{T}$ must be of the form $\left(\ell_{V}^{T}, \ell_{Y}^{T}\right)$, where $\ell_{V}$ is the unique unstable eigenvector, associated with eigenvalue $\alpha$, of the purely gas-dynamical matrix $f_{V-}^{0}\left(f_{V-}^{1}\right)^{-1}$, and

$$
\ell_{Y}^{T}=\ell_{V}^{T}\left(\lambda f_{V-}^{0}\left(f_{V-}^{1}\right)^{-1} f_{Y-}^{1}+Q K \psi_{-}\right)\left(\lambda\left(g_{-}^{0}-\alpha g_{-}^{1}\right) I+K \psi_{-}\right)^{-1} .
$$

To determine $\alpha, \ell_{V}$, and thereby $\ell_{Y}$, we observe that $f_{V-}^{0}\left(f_{V-}^{1}\right)^{-1}$ is related by similarity transform $M \rightarrow\left(f_{V-}^{0}\right)^{-1} M f_{V-}^{0}$ to the inverse $\left(f_{V-}^{1}\right)^{-1} f_{V-}^{0}$ of the hyperbolic convection matrix $\left(f_{V}^{0}\right)^{-1} f_{V}^{1}$ of the nonreactive Euler equations $V_{t}+\left(f_{V}^{0}\right)^{-1} f_{V}^{1} V_{x}=0$ written in nonconservative form in $V$ coordinates with $Y \equiv 0$. Thus, $\alpha^{-1}$ is an eigenvalue of $\left(f_{V}^{0}\right)^{-1} f_{V}^{1}$, i.e., a hyperbolic characteristic speed of the nonreactive Euler equations, and $\ell_{V}^{T}=\tilde{\ell}^{T}\left(f_{V-}^{0}\right)^{-1}$, where $\tilde{\ell}$ is the associated left characteristic direction (eigenmode).

Noting that $\alpha^{-1}$, as the unique positive characteristic at state $V=V_{-}$, must be the largest characteristic speed, we have by standard formulae [46, 42, 43, 35, 51] or direct calculation

$$
\ell_{V}^{T}=\tilde{\ell}^{T}\left(f_{V-}^{0}\right)^{-1}=\left(p_{\rho}-c u+\rho^{-1} p_{e}\left(u^{2} / 2-e\right), c-\rho^{-1} p_{e} u, \rho^{-1} p_{e}\right),
$$


determining $\ell^{T}(\lambda)=\left(\ell_{V}^{T}, \ell_{Y}^{T}\right)(\lambda)$ through A.2). Note that $\ell_{V}$ is independent of $\lambda$. For $Y$ independent equations of state, (A.2) simplifies considerably, to $\ell_{Y}^{T}=\ell_{V}^{T} Q K \psi_{-}\left(\lambda\left(g_{-}^{0}-\right.\right.$ $\left.\left.\alpha g_{-}^{1}\right) I+K \psi_{-}\right)^{-1}$.

REMARK A.1. Noting that the $e$-component $\rho^{-1} p_{e}$ of $\ell_{V}$ does not vanish in (A.3), we may alternatively rescale by $\rho / p_{e}$ to obtain an analytic choice of the form $\ell^{T}=(*, *, 1, *)$ convenient for numerical solution.

A.1. Alternative, numerical computation. Alternatively, an analytic choice of $\ell$ may be determined numerically by solution of Kato's ODE [31] as described in [16, 28, 53, 54]. For $\Re \lambda$ bounded from zero, this involves finding numerically at each $\lambda$-value the unique stable left and right eigenvectors of $G_{-}$and computing the associated eigenprojection for use in the Kato ODE as in the general problem-independent method of [16, 28, [53, 54. At or near $\Re \lambda=0$, however, this method must be modified, since the stable eigenvector becomes neutral at $\Re \lambda=0$. A simple resolution is to notice that, there, the eigenvalues of $G_{-}$consist of a single eigenvalue with strictly positive real part, which may be discarded, and three eigenvalues of the form $g_{j}=\alpha_{j} \lambda$, where $\alpha_{j}$ (see above) are hyperbolic characteristic speeds for the nonreactive Euler equations, of which the one for which $g_{j} / \lambda=\alpha_{j}<0$ is the one associated with $\ell$.

Appendix B. Ideal gas profile. In this appendix, we explicitly solve (2.12) for the case of an ideal gas. Restricting to a steady shock, $s=0$, and using the ideal gas law (2.2), we may rewrite (2.12) as

$$
\begin{aligned}
\bar{\rho} \bar{u} & =\rho_{ \pm} u_{ \pm}:=-m, \\
\bar{u}+\Gamma \frac{\bar{e}}{\bar{u}} & =u_{ \pm}+\Gamma \frac{e_{ \pm}}{u_{ \pm}}:=b, \\
\frac{\bar{u}^{2}}{2}+(\Gamma+1) \bar{e}+q \bar{Y} & =\frac{u_{ \pm}^{2}}{2}+(\Gamma+1) e_{ \pm}+q Y_{ \pm}:=c .
\end{aligned}
$$

Combining the second two equations and simplifying gives $(\Gamma+2) \bar{u}^{2}-2(\Gamma+1) b \bar{u}+2 \Gamma(c-$ $q \bar{Y})=0$. Solving using the quadratic formula, we obtain

$$
\bar{\rho}=-\frac{m}{\bar{u}}, \quad \bar{e}=\frac{b \bar{u}-\bar{u}^{2}}{\Gamma}, \quad \bar{u}=\frac{\Gamma+1}{\Gamma+2} b \pm \sqrt{\left(\frac{\Gamma+1}{\Gamma+2}\right)^{2} b^{2}+\frac{2 \Gamma(q \bar{Y}-c)}{\Gamma+2}}
$$

where we have chosen the negative solution branch for $\bar{u}$ in accordance with the fact that $[u]>0$, or, equivalently, $[\rho]<0$, for a right-moving gas-dynamical shock, so that $\bar{u}\left(0^{-}\right)<u_{+}$. (Recall that $\bar{u}\left(0^{-}\right)$and $u_{+}$are the two branches of the square root for $Y=1$, corresponding to the solutions of the Rankine-Hugoniot conditions for a nonreacting gas-dynamical shock.) With (5.3), (B.2) gives an explicit expression for the profile as a function of the variable $y$.

For a given Neumann shock, there is a one-parameter family of possible endstates $(\rho, u, e)_{-}$determined by the value of $q$, the maximum value of $q$ corresponding to a Chapman-Jouget wave, for which the argument of the square root vanishes for $y=0$. 


\section{REFERENCES}

[1] J. Alexander, R. Gardner and C.K.R.T. Jones. A topological invariant arising in the analysis of traveling waves. J. Reine Angew. Math. 410 (1990) 167-212. MR.1068805 (92d:58028)

[2] G. Abouseif and T.Y. Toong. Theory of unstable one-dimensional detonations, Combust. Flame 45 (1982) 67-94.

[3] S. Alinhac. Existence d'ondes de raréfaction pour des systèmes quasi-linéaires hyperboliques multidimensionnels. Comm. Partial Differential Equations, 14(2):173-230, 1989. MR976971 (90h:35147b)

[4] B. Barker, J. Humpherys, K. Rudd, and K. Zumbrun. Stability of viscous shocks in isentropic gas dynamics, Comm. Math. Phys. 281 (2008), no. 1, 231-249. MR2403609 (2009c:35286)

[5] B. Barker, J. Humpherys, and K. Zumbrun. One-dimensional stability of parallel shock layers in isentropic magnetohydrodynamics, J. Differential Equations, 249(9):2175-2213, 2010. MR2718655 (2011j:76088)

[6] B. Barker, J. Humpherys, and K. Zumbrun. STABLAB: A MATLAB-based numerical library for Evans function computation. Available at: http://impact.byu.edu/stablab/.

[7] B. Barker, O. Lafitte, and K. Zumbrun. Existence and stability of viscous shock profiles for 2-D isentropic MHD with infinite electrical resistivity. Acta Math. Sci. Ser. B Engl. Ed., 30(2):447-498, 2010. MR2656550 (2011c:35391)

[8] B. Barker, M. Lewicka, and K. Zumbrun. Existence and stability of viscoelastic shock profiles, Arch. Ration. Mech. Anal. 200 (2011), 491-532. MR2787588

[9] B. Barker, S. Shaw, S. Yarahmadian, and K. Zumbrun. Existence and stability of steady states of a reaction convection diffusion equation modeling microtubule formation, to appear, J. Math. Biology.

[10] B. Barker and K. Zumbrun. Numerical stability of ZND detonations for Majda's model, preprint (2010).

[11] B. Barker and K. Zumbrun. Numerical stability of ZND detonations, in preparation.

[12] A. Bourlioux, A. Majda, and V. Roytburd. Theoretical and numerical structure for unstable onedimensional detonations. SIAM J. Appl. Math. 51 (1991) 303-343. MR.1095022 (91m:76090)

[13] T. J. Bridges, G. Derks, and G. Gottwald. Stability and instability of solitary waves of the fifth-order KdV equation: a numerical framework. Phys. D, 172(1-4):190-216, 2002. MR 1946769 (2004i:37148)

[14] L. Q. Brin. Numerical testing of the stability of viscous shock waves. Ph.D. thesis, Indiana University, Bloomington, 1998. MR.2697543

[15] L. Q. Brin. Numerical testing of the stability of viscous shock waves. Math. Comp., 70(235):10711088, 2001. MR1710652 (2001j:65118)

[16] L. Q. Brin and K. Zumbrun. Analytically varying eigenvectors and the stability of viscous shock waves. Mat. Contemp., 22:19-32, 2002. Seventh Workshop on Partial Differential Equations, Part I (Rio de Janeiro, 2001). MR.1965784 (2004c:15012)

[17] J. Buckmaster and J. Neves. One-dimensional detonation stability: the spectrum for infinite activation energy. Phys. Fluids 31 (1988) no. 12, 3572-3576.

[18] N. Costanzino, J. Humpherys, T. Nguyen, and K. Zumbrun. Spectral stability of noncharacteristic isentropic Navier-Stokes boundary layers. Arch. Ration. Mech. Anal. 192 (2009), no. 3, 537-587. MR 2505363

[19] J. J. Erpenbeck. Stability of step shocks. Phys. Fluids, 5:1181-1187, 1962. MR0155515 (27:5449)

[20] J. J. Erpenbeck. Stability of steady-state equilibrium detonations. Physics of Fluids, 5(5):604-614, 1962.

[21] J. J. Erpenbeck. Stability of idealized one-reaction detonations, Phys. Fluids, 7 (1964).

[22] W. Fickett and W. C. Davis. Detonation: Theory and Experiment. Dover Publications, 2000.

[23] W. Fickett and W. Wood. Flow calculations for pulsating one-dimensional detonations. Phys. Fluids 9 (1966) 903-916.

[24] G. R. Fowles. On the evolutionary condition for stationary plane waves in inert and reactive substances. In Shock induced transitions and phase structures in general media, volume 52 of IMA Vol. Math. Appl., pages 93-110. Springer, New York, 1993. MR.1240334 (94g:76043)

[25] R. A. Gardner and K. Zumbrun. The gap lemma and geometric criteria for instability of viscous shock profiles. Comm. Pure Appl. Math., 51(7):797-855, 1998. MR1617251 (99c:35152)

[26] J. Humpherys, O. Lafitte, and K. Zumbrun. Stability of isentropic Navier-Stokes shocks in the high-Mach number limit. Comm. Math. Phys., 293(1):1-36, 2010. MR2563797(2010i:76085) 
[27] J. Humpherys, G. Lyng, and K. Zumbrun. Spectral stability of ideal-gas shock layers. Arch. Ration. Mech. Anal., 194(3):1029-1079, 2009. MR2563632 (2011b:35329)

[28] J. Humpherys and K. Zumbrun. An efficient shooting algorithm for Evans function calculations in large systems. Phys. D, 220(2):116-126, 2006. MR2253406 (2007e:35006)

[29] H. K. Jenssen, G. Lyng, and M. Williams. Equivalence of low-frequency stability conditions for multidimensional detonations in three models of combustion. Indiana Univ. Math. J., 54(1):1-64, 2005. MR2126075 (2006a:35249)

[30] T. Kapitula and B. Sandstede. Stability of bright solitary-wave solutions to perturbed nonlinear Schrödinger equations. Phys. D, 124(1-3):58-103, 1998. MR1662530 (99h:35199)

[31] T. Kato, Perturbation theory for linear operators. Springer-Verlag, Berlin, Heidelberg, 1976. MR0407617 (53:11389)

[32] A.R. Kasimov and D.S. Stewart. Spinning instability of gaseous detonations. J. Fluid Mech. 466 (2002), 179-203. MR1925152 (2003g:76093)

[33] O. Lafitte, M. Williams, and K. Zumbrun. High-frequency asymptotics and multi-d instability of ZND detonations. In preparation.

[34] H. I. Lee and D. S. Stewart. Calculation of linear detonation instability: one-dimensional instability of plane detonation. J. Fluid Mech., 216:103-132, 1990.

[35] G. Lyng and K. Zumbrun. One-dimensional stability of viscous strong detonation waves. Arch. Ration. Mech. Anal., 173(2):213-277, 2004. MR2081031 (2005f:76061)

[36] G. Lyng and K. Zumbrun. A stability index for detonation waves in Majda's model for reacting flow. Phys. D, 194(1-2):1-29, 2004. MR2075662 (2005d:35134)

[37] A. Majda. The stability of multidimensional shock fronts. Mem. Amer. Math. Soc., 41(275):iv+95, 1983. MR683422 (84e:35100)

[38] C. Mascia and K. Zumbrun. Stability of large-amplitude viscous shock profiles of hyperbolicparabolic systems. Arch. Ration. Mech. Anal., 172(1):93-131, 2004. MR2048568 (2005d:35166)

[39] R. L. Pego and M. I. Weinstein. Eigenvalues, and instabilities of solitary waves. Philos. Trans. Roy. Soc. London Ser. A, 340(1656):47-94, 1992. MR1177566 (93g:35115)

[40] B. Sandstede. Stability of travelling waves. In Handbook of dynamical systems, Vol. 2, pages 9831055. North-Holland, Amsterdam, 2002. MR1901069 (2004e:37121)

[41] B. Sandstede. private communication, 1999.

[42] D. Serre. Systems of conservation laws. 1. Cambridge University Press, Cambridge, 1999. Hyperbolicity, entropies, shock waves, Translated from the 1996 French original by I. N. Sneddon. MR1707279 (2000g:35142)

[43] D. Serre. Systems of conservation laws. 2. Cambridge University Press, Cambridge, 2000. Geometric structures, oscillations, and initial-boundary value problems, Translated from the 1996 French original by I. N. Sneddon. MR1775057 (2001c:35146)

[44] M. Short and D. S. Stewart. The multi-dimensional stability of weak-heat-release detonations. $J$. Fluid Mech., 382:109-135, 1999. MR1680620(99m:80017)

[45] D. S. Stewart and A. R. Kasimov, On the State of Detonation Stability Theory and Its Application to Propulsion, Journal of Propulsion and Power, 22:6, 1230-1244, 2006.

[46] J. Smoller. Shock waves and reaction-diffusion equations. Springer-Verlag, New York, second edition, 1994. MR 1301779 (95g:35002)

[47] B. Texier and K. Zumbrun. Galloping instability of viscous shock waves. Phys. D, 237(10-12):15531601, 2008. MR2454606 (2009h:35271)

[48] B. Texier and K. Zumbrun. Hopf bifurcation of viscous shock waves in compressible gas dynamics and MHD. Arch. Ration. Mech. Anal., 190(1):107-140, 2008. MR2434902 (2009g:35239)

[49] B. Texier and K. Zumbrun. Transition to longitudinal instability of detonation waves is generically associated with Hopf bifurcation to time-periodic galloping solutions, Comm. Math. Physics, 302:151, 2011. MR2770009

[50] K. Zumbrun. Multidimensional stability of planar viscous shock waves. In Advances in the theory of shock waves, volume 47 of Progr. Nonlinear Differential Equations Appl., pages 307-516. Birkhäuser Boston, Boston, MA, 2001. MR 1842778 (2002k:35200)

[51] K. Zumbrun. Stability of large-amplitude shock waves of compressible Navier-Stokes equations. In Handbook of mathematical fluid dynamics. Vol. III, pages 311-533. North-Holland, Amsterdam, 2004. With an appendix by Helge Kristian Jenssen and Gregory Lyng. MR2099037 (2006f:35229)

[52] K. Zumbrun. Stability of detonation profiles in the ZND limit. Arch. Rational Mech. Anal., 200:141182, 2011. MR 2781588 
[53] K. Zumbrun. A local greedy algorithm and higher-order extensions for global numerical continuation of analytically varying subspaces. Quart. Appl. Math., 68:557-561, 2010. MR2676976|(2011e:65124)

[54] K. Zumbrun. Numerical error analysis for Evans function computations: a numerical gap lemma, centered-coordinate methods, and the unreasonable effectiveness of continuous orthogonalization. preprint, 2009.

[55] K. Zumbrun. High-frequency asymptotics and stability of ZND detonations in the high-overdrive and small-heat release limits. to appear, Arch. Ration. Mech. Anal. 